\title{
ENGEVISTA
}

Página da revista: http://www.uff.br/engevista/seer/

\section{Projeto e desenvolvimento de módulo de controle de nível em escala piloto}

\author{
Felipe Gnoatto ${ }^{1}$ \\ Elciane Regina Zanatta ${ }^{2}$ \\ Dirceu de Melo $^{3}$ \\ Yuri Ferruzzi ${ }^{4}$ \\ Estor Gnoattto 5
}

Resumo: Processos industriais geralmente necessitam de algum tipo de controle, podendo ser manual ou automatizado. O controle é feito em alguns parâmetros como vazão, temperatura, concentração, e controle de nível. O controle de fluxo é mais utilizado nas saídas ou entradas de tanques, controlando a concentração exigida naquele recipiente. Este trabalho teve como objetivo desenvolver um módulo de controle de nível em escala piloto, que poderá realizar o controle de maneira automática, e verificar a reprodutibilidade dos sensores e atuadores com a intenção de fixar o conhecimento já adquirido e disponibiliza-lo para ser utilizado na formação acadêmica profissional de outros estudantes de engenharia. O módulo foi projetado, construído e instalado, e foram realizadas curvas de calibração para os sensores de vazão e os sensores de pressão, e foi analisada a vazão máxima para a operação do módulo. Obteve-se como resultado uma dependência linear entre a tensão $(\mathrm{mV})$ e a altura da coluna d'água do módulo. Observou-se uma dependência linear entre a frequência (Hz) e a vazão de líquido do módulo. A vazão máxima de operação determinada experimentalmente para o módulo desenvolvido é de 7,3 L.min ${ }^{-1}$. As calibrações dos sensores de pressão e de fluxo demonstraram que todos os sensores apresentam reprodutibilidade na faixa de operação a ser usada.

Palavras-chave: Módulo experimental, Vazão, Nível, Calibração.

\footnotetext{
${ }^{1}$ Unioeste - Universidade Estadual do Oeste do Paraná - Campus de Toledo

${ }^{2}$ UTFPR - Universidade Tecnológica Federal do Paraná - Campus de Medianeira

${ }^{3}$ UTFPR - Universidade Tecnológica Federal do Paraná - Campus de Medianeira

${ }^{4}$ UTFPR - Universidade Tecnológica Federal do Paraná - Campus de Medianeira

${ }^{5}$ UTFPR - Universidade Tecnológica Federal do Paraná - Campus de Medianeira
}

ENGEVISTA, V. 18, n. 2, p. 280-293, Dezembro 2016. 
ISSN: $1415-7314$

ISSN online: 2317-6717

Abstract: Industrial processes require usually some kind of control, that can be automated or manual. The control is done on some parameters such as flow, temperature, concentration, and level control. Flow control is most used on the outputs or inputs tanks controlling the concentration required in the container. This study aimed to develop a level control module in pilot scale that could make the control automatically, and check the reproducibility of sensors and actuators with the intention of fixing the existing knowledge and make it available for use in professional academic training other engineering students. The module was designed, constructed and installed, and calibration curves for flow sensors and pressure sensors were performed, and we analyzed the maximum flow for the operation of the module. Obtained as a result a linear dependence between the voltage $(\mathrm{mV})$ and the height of the water column of the module. There was a linear dependence between the frequency $(\mathrm{Hz})$ and the liquid flow module. The maximum flow ratio determined experimentally developed for the module is $7.3 \mathrm{~L} \cdot \mathrm{min}^{-1}$. The calibration of the pressure and flow sensors showed that all of the sensors have reproducibility in the operating range to be used.

Keywords: Experimental module, Flow, Level, Calibration. 
ISSN: $1415-7314$

ISSN online: 2317-6717

\section{Introdução}

O controle de processos está presente nas atividades de qualquer profissional, especificamente no cotidiano dos engenheiros. Inúmeros equipamentos requerem a necessidade de algum tipo de domínio. Os diversos processos necessitam de controles efetuados por parâmetros, que podem ser de vazão, temperatura, nível, concentração entre outros, que são feitos de maneira manual por meio de operador ou de maneira automatizada através de controladores (Senai, 1999a).

Para o pesquisador Schmidt, (2008) o controle de nível é importante em várias situações industriais, onde os níveis de líquido devem ser mantidos numa altura desejada. Na Indústria, muitas vezes, é necessário um controle preciso de nível, há misturas de componentes, que requerem porções exatas, para o melhor resultado das reações. Se forem efetuadas de maneira correta preservando os valores necessários à operação atingirá o objetivo final da reação.

O controle de fluxo é mais utilizado nas saídas ou entradas de tanques controlando a concentração exigida naquele recipiente. Podemos ter também o controle da vazão, causada por duas correntes com temperaturas diferentes, que se misturam para ter uma terceira temperatura (Mathias, 2008).

A principal função de um sistema de controle é regular as variáveis, deixando-as em valores constantes pré-fixados. A regulagem deve ser feita quando há variação no ponto de ajuste, essa alteração não ocorre com muita frequência e são usualmente menores que $10 \%$ do fundo de escala. Outro momento em que é preciso a intervenção é quando ocorrem distúrbios. Se o ponto de ajuste for mudado, a variável controlada sofrerá uma alteração também. A variação de saída normalmente é lenta, da ordem de minutos ou horas (Ribeiro, 2001).

Segundo o pesquisador Pinto (2005), nas últimas décadas, começou-se a dispor de computadores e microprocessadores que se tornaram a base da tecnologia de automação da forma que conhecemos atualmente. Sabe-se que sem o auxilio da tecnologia seria difícil montar e desenvolver sistemas de controle em tempo real.

Os sistemas trabalham com controle realimentado com constante medição da variável controlada, permitindo a visualização e correção de possíveis erros causados nas variáveis que podem ser manipuladas (Albernaz et al, 2012).

Nos últimos 20 anos adotaram-se cada vez mais os computadores gerando uma inovação na instrumentação, medição e automação. Consequentemente surge a instrumentação virtual, esta utiliza o computador em conjunto com programas, sensores e controladores, possibilitando a construção de sistemas que se adaptam as necessidades do usuário ao invés de estarem limitadas as predefinições de sistemas tradicionais (Neto e Nascimento, 2007).

Para Neto e Nascimento (2007) a programação do sistema de controle pode ser feita por diferentes tipos de programas, estes pesquisadores citam dois, o primeiro software Simulink ${ }^{\circledR}$ que é uma ferramenta inclusa no MATLAB feito pela MathWorks, o segundo software é o LabVIEW sendo que este tem uma linguagem de programação completa, que é comparável com outras linguagens de

ENGEVISTA, V. 18, n. 2, p. 280-293, Dezembro 2016. 
ISSN: $1415-7314$

ISSN online: 2317-6717

programação como $\mathrm{C}, \mathrm{C}++$ ou Visual Basic, estes pesquisadores concluem que é possível usar ambos pois há a possibilidade de interface entre os mesmos.

Conforme Senai (1999a), o controle pode ocorrer em malha aberta ou em malha fechada, no primeiro a ação de controle é realizada sem a análise da saída, não há a verificação se o controle foi efetivo ou não. No segundo a ação de controle é influenciada de algum jeito pela saída, e a saída é constantemente analisada e comparada para diminuir o erro e mantê-la no valor desejado.

Quando se tem a combinação do controle proporcional, integral e derivativo tem-se o controle Proporcional Integral Derivativo (PID), que possui muitas variações, três delas são a forma paralela, em série e expandida do controle PID (Seborg et al, 2004).

Para a empresa de automação Smar (2011), na indústria há a necessidade de se saber o nível de fluídos e sólidos é importante para a operação de processo em si, bem como para cálculo de custo. Nível é a altura do conteúdo que há dentro de um reservatório podendo ser este um líquido ou um sólido. A medição de nível pode ser direta, indireta e medição descontinua.

Os sensores de níveis podem ser divididos em dois grupos principais os de contato que entram em contato direto com o fluido e os sem contato que tem contado direto com o meio. E dentro desses dois grupos os sensores podem fazer uma medição direta neste caso podem-se usar visores de nível boia ou flutuador. Ou se faz uma medição indireta que pode ser feita através de medidores capacitivos de pressão, ultrassom entre outros (Bojorge, 2014).

Os sensores de vazão podem ser medidores indiretos, que fazem o uso de fenômenos ligados à quantidade de fluido escoando, sendo os mais comuns são os tubo de Pitot, tubo de Venturi, rotâmetro e placa de orifício. Podem fazer a leitura de maneira direta, sendo neste caso os mais comuns os medidor tipo turbina, tipo hélice e calhas Parshall (Senai, 1999b).

Neste trabalho foi utilizado um medidor tipo turbina. O medidor de vazão tipo turbina é um medidor de vazão para líquidos e gases limpos a medição é feita através do número de voltas que a turbina dá em um determinado período. É amplamente utilizada pela sua alta confiabilidade, funcionando muito bem com regime turbulento. Deve-se tomar cuidado, pois uma turbina calibrada para operar com água não poderá ser utilizada para medir a vazão de outro fluido (Ribeiro, 2003).

O servo-motor é amplamente utilizado em robótica e modelismo. Ele pode variar a posição de seu eixo de 0 -180 graus, o ângulo do eixo depende da largura de pulso que é dado pelo controlador (PWM- Pulse Width Modulation), normalmente esse tempo de pulso varia de $1 \mathrm{~ms}$ até $2 \mathrm{~ms}$. Se for mandado um pulso de 1,5 ms o motor ficará na metade de sua posição (90 graus) (Santos, 2007).

O objetivo deste trabalho foi projetar e desenvolver um módulo de controle de processo de nível em escala piloto, e verificar a reprodutibilidade dos sensores e atuadores com a intenção de disponibilizar este módulo para ser utilizado em pesquisas e na formação acadêmica profissional de outros estudantes de engenharia. 


\section{Seção experimental}

\section{Projeto e montagem do módulo}

Na Figura 1, é apresentado o projeto do módulo de controle de nível. A estrutura metálica do módulo, indicada na Figura 1, como (01), foi montada com tubo industrial 30 x 30 mm, SAE 1020, sobre uma base móvel de rodízios de pluoretano tendo altura vertical de aproximadamente $2 \mathrm{~m}$.

O módulo também é composto por dois tanques de PVC (02) de $200 \mathrm{~mm}$ de diâmetro e 900 $\mathrm{mm}$ de altura, interligados por tubos de $1 / 2$ polegada (03), o primeiro tanque é alimentado com água por meio de uma bomba (04). Ambos tem medidores de pressão no fundo, válvulas proporcionais de fluxo na saída, e medidores de vazão na entrada e saída dos tanques (05).

Na Figura 2, são apresentados detalhes do módulo projetado que também é composto por quatro válvulas do tipo esfera (06), e duas válvulas proporcionais elétricas, (07).

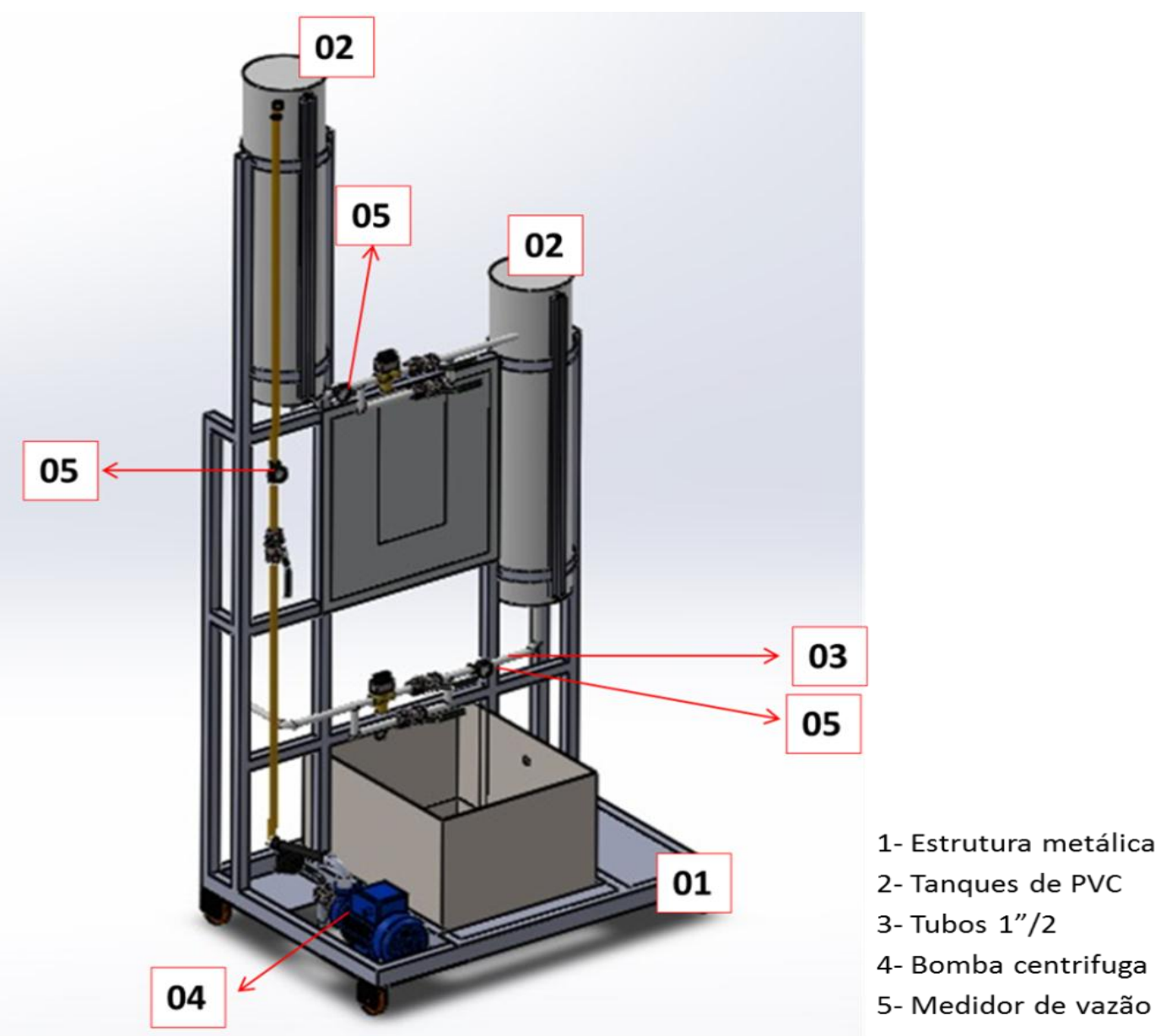

Figura 1. Projeto do módulo experimental de controle de nível. 


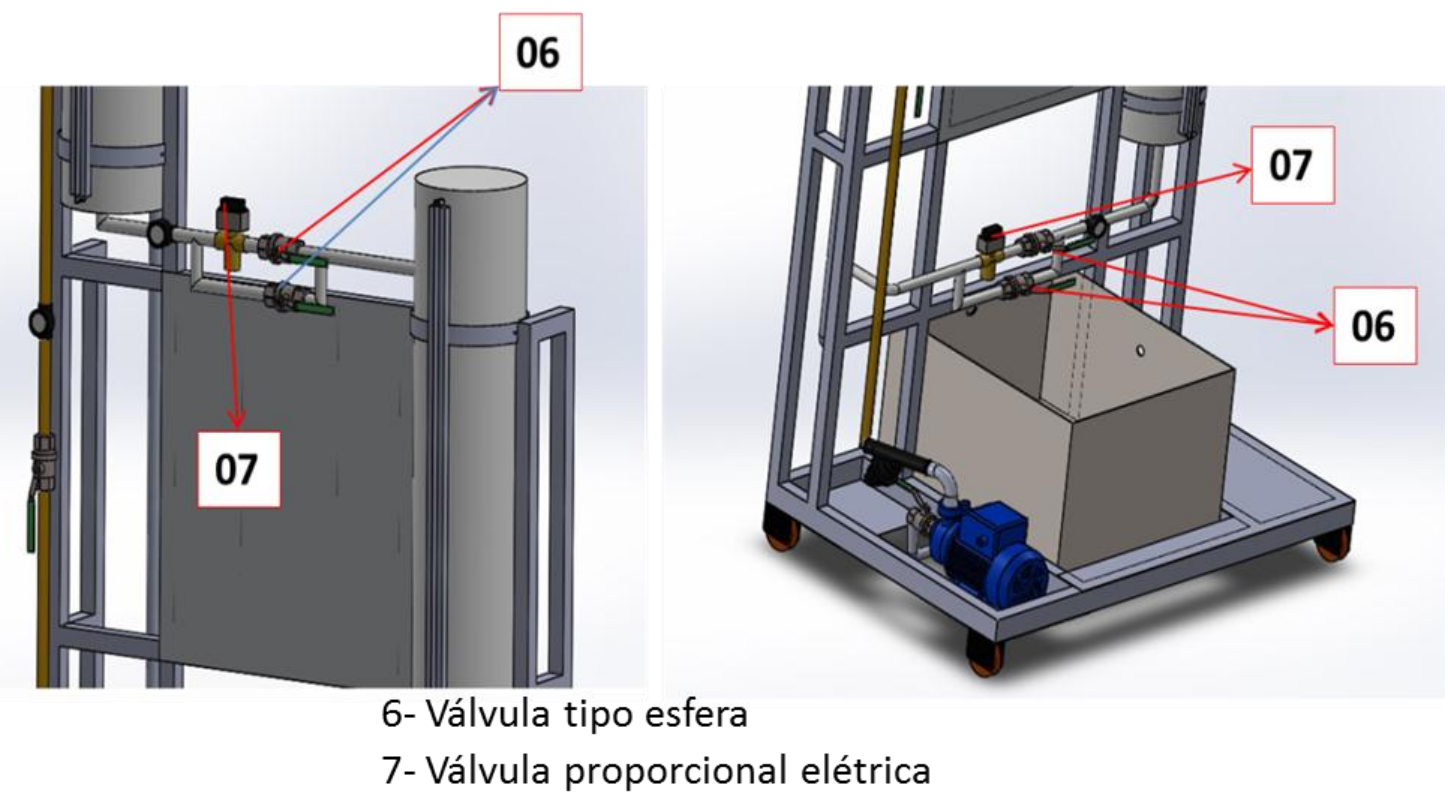

Figura 2. Detalhes do projeto do módulo experimental de controle de nível.

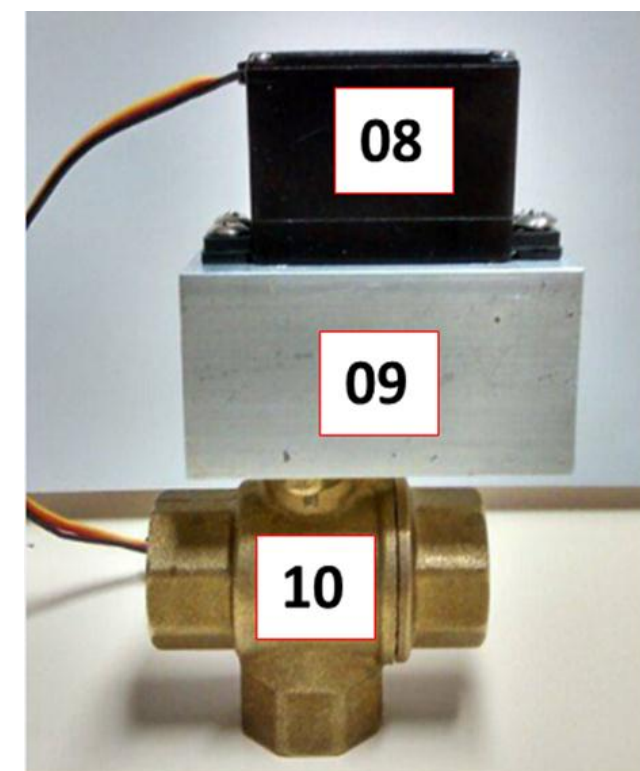

\section{8- Servo motor 9- Encaixe} 10- Válvula esfera

Figura 3. Servo motor, encaixe e válvula proporcional elétrica, utilizado na montagem do módulo experimental de controle de nível.

Na Figura 3 podemos observar o servo-motor (08), o encaixe (09) e a válvula (10). Optou-se por adaptar um servo-motor a essa base, que já possuía um lugar para a fixação do motor. O encaixe utilizado para acoplar a válvula, foi confeccionado também para transmitir o torque do motor para a válvula. A válvula proporcional elétrica, foi confeccionada a partir de uma válvula elétrica do tipo on-off, modelo de esfera. Os sensores que foram colocados nos tanques do módulo são de contato e de medição indireta, feita através da pressão. Podemos visualizar o sensor na Figura 4. 


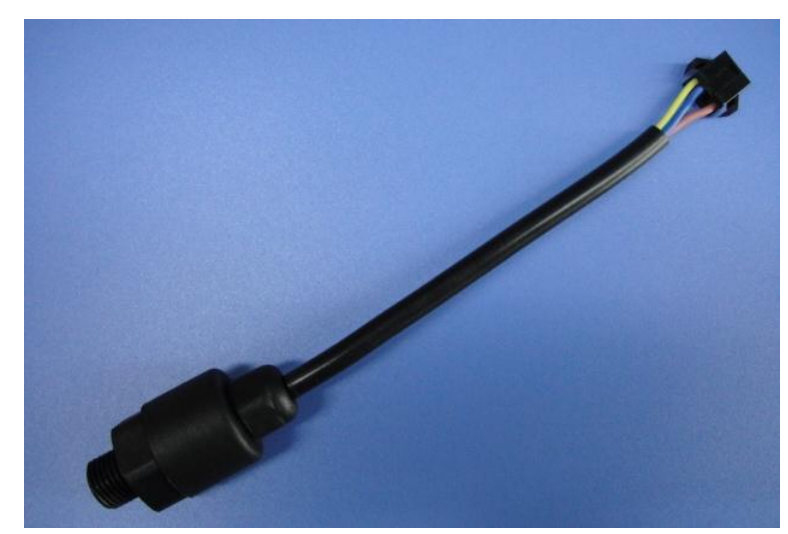

Figura 4. Sensor de pressão utilizado na montagem do módulo experimental de controle de nível.

O sensor de pressão é modelo HM4100b da marca Ever-Smart, e apresenta efetividade de 04 bar, erro de $3 \%$. A pressão $P$, pode ser calculada pela Equação 01.

$$
T_{s}=0,2 \times T_{a}(P+0,5)
$$

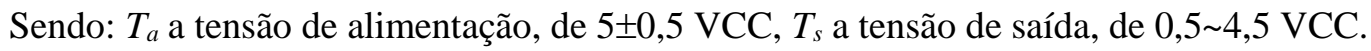

O sensor dará como resultado uma pressão e com ela pode-se calcular através da Equação 02 a altura de líquido $h$ em cm, sendo necessário conhecer como informação adicional a densidade $\rho$ em g.cm ${ }^{-3}$ e a aceleração da gravidade $g$ de $9,80665 \mathrm{~m} \cdot \mathrm{s}^{-2}$.

$$
h=\frac{P}{\rho * g}
$$

Os medidores de vazão utilizados, foram os da marca $S e^{a "}$, e modelo YF-S201, como mostrado na Figura 5, onde seu tamanho é comparado a de uma moeda.

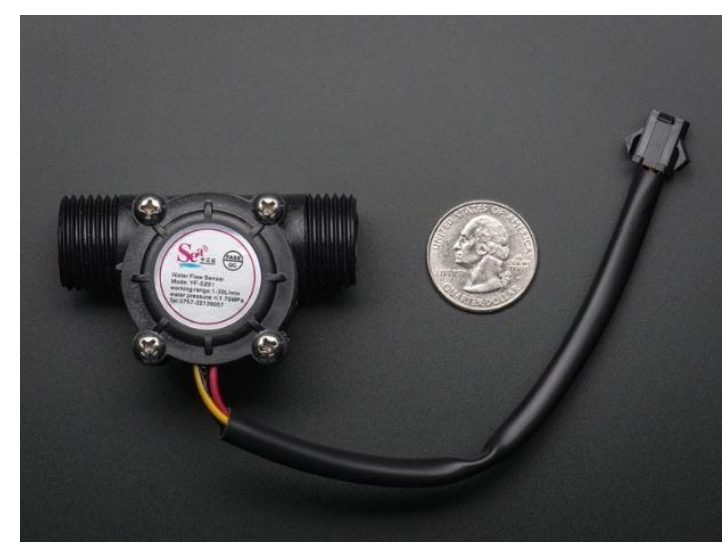

Figura 5. Medidor de vazão YF-S201 utilizados na montagem do módulo experimental de controle de nível.

Os dados técnicos sensor YF-S201 são vazão de 1 - $30 \mathrm{~L} \cdot \mathrm{min}^{-1}$, pulsos por litro de 450 e a frequência em função da vazão é dado pela Equação 03: 


$$
F=7,5 \times Q
$$

Sendo: $F$ a frequência em Hz, e $Q$ a vazão em L.min ${ }^{-1}$.

\section{Analise dos equipamentos}

Análise de cada equipamento medidor de pressão e vazão foi realizada separadamente.

Foram feitas as curvas de calibração para os sensores de pressão e de vazão.

Para o sensor de pressão foi feito uma curva da altura de liquido $(\mathrm{cm})$ versus a tensão de saída do sensor $(\mathrm{mV})$. Com os dados obtidos experimentalmente foi gerada a equação de calibração do sensor de pressão.

Com a tensão de saída do sensor para cada ponto experimental, a Equação 01 foi usada para calcular a pressão, e logo em seguido usou-se a Equação 02 para calcular a altura de líquido no tanque dada pela equação fornecida pelo fabricante.

Para o sensor de fluxo fez-se uma curva da frequência $(\mathrm{Hz})$ versus a vazão $\left(\mathrm{L}_{\text {. }} \mathrm{min}^{-1}\right)$. Com os dados obtidos experimentalmente foi gerada a equação de calibração do sensor de vazão.

Com a frequência $(\mathrm{Hz})$ de pulsos obtidos experimentalmente, foi usado a Equação 03, para calcular a vazão de líquido dada pela equação fornecida pelo fabricante.

E para a válvula elétrica fez-se um gráfico de vazão $\left(\right.$ L. $\left.\mathrm{min}^{-1}\right)$ versus o ângulo de abertura.

Para medição dos dados de vazão foi utilizado o sensor de fluxo da Contech, que possui laudo de calibração. E para a aquisição dos dados de todos os sensores foi usado o modelo FieldLogger da marca NOVUS. Com a análise de cada equipamento separadamente foi possível calibrar e assim disponibilizar o módulo experimental para as aulas práticas e as pesquisas de forma reprodutiva e confiável.

\section{Resultados e discussão}

\section{Apresentação do módulo executado}

Na Figura 6 é apresento o módulo de controle de nível pronto e instalado no laboratório de engenharia química III, da Universidade Estadual do Oeste do Paraná - Campus de Toledo. 


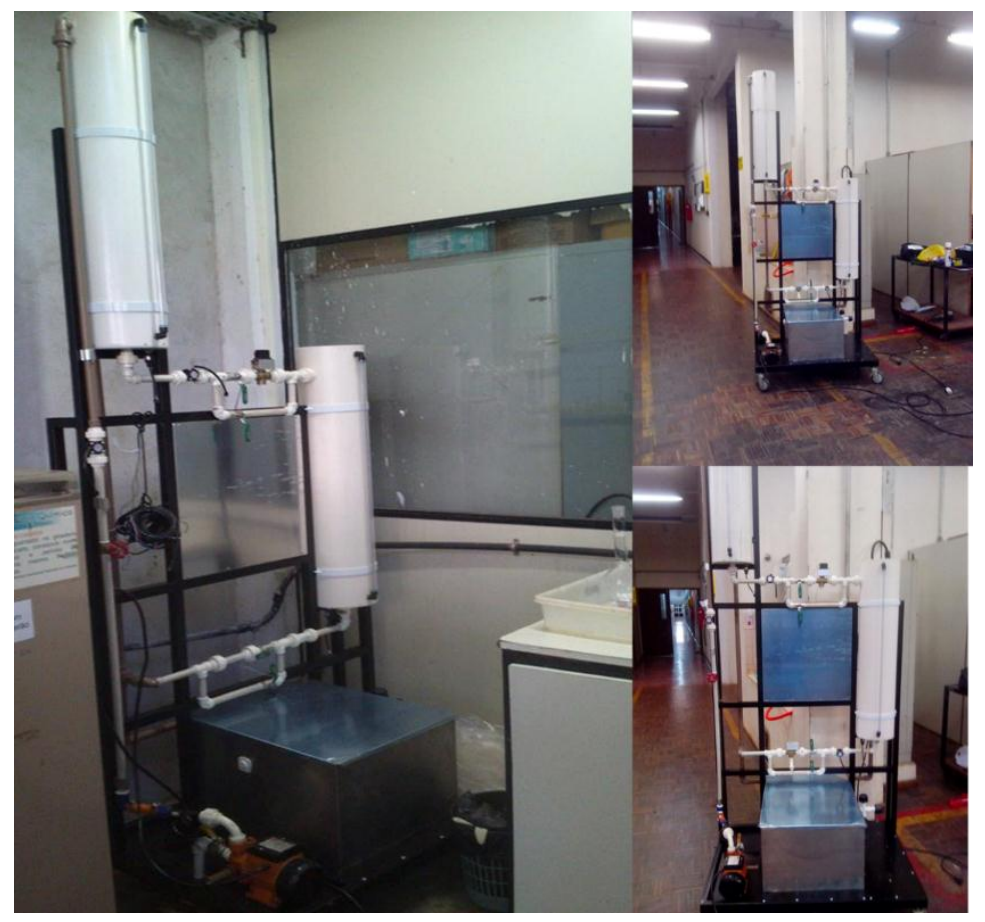

Figura 6. Módulo de controle de nível pronto, instalado no laboratório de engenharia química III da Universidade Estadual do Oeste do Paraná - Campus de Toledo.

\section{Determinação da curva de calibração do sensor de pressão}

$\mathrm{Na}$ determinação da curva de calibração do sensor de pressão obteve-se os dados apresentados na Tabela 1. Os valores foram obtidos para dezoito pontos experimentais em que a pressão foi variada entre $83-0$ cm.c.a.

Tabela 1. Determinação curva de calibração para o sensor de pressão instalado no módulo experimental de controle de nível.

\begin{tabular}{cccc}
\hline $\begin{array}{c}\text { Altura de água medida } \\
(\mathbf{c m})\end{array}$ & $\begin{array}{c}\text { Altura Calculada * } \\
(\mathbf{c m})\end{array}$ & $\begin{array}{c}\text { Tensão } \\
(\mathbf{m V})\end{array}$ & $\begin{array}{c}\text { Desvio padrão } \\
\text { entre as alturas }\end{array}$ \\
\hline 83 & 94,347 & 592,23 & 8,023 \\
80 & 91,786 & 589,73 & 8,334 \\
75 & 87,070 & 585,12 & 8,535 \\
70 & 82,300 & 580,45 & 8,698 \\
65 & 77,379 & 575,64 & 8,753 \\
60 & 72,745 & 571,11 & 9,012 \\
55 & 67,731 & 566,21 & 9,002 \\
50 & 63,250 & 561,83 & 9,369 \\
45 & 57,121 & 555,84 & 8,571 \\
40 & 51,974 & 550,81 & 8,467 \\
35 & 47,210 & 546,15 & 8,634 \\
30 & 42,309 & 541,36 & 8,704 \\
25 & 37,382 & 536,54 & 8,756 \\
20 & 32,336 & 531,61 & 8,723 \\
15 & 27,240 & 526,63 & 8,655 \\
10 & 20,292 & 519,84 & 7,277 \\
\hline
\end{tabular}




\begin{tabular}{llll}
\hline 5 & 15,255 & 514,91 & 7,252 \\
0 & 11,673 & 511,41 & 8,254 \\
\hline
\end{tabular}

* Calculado através da Equação 02.

O sensor de pressão foi alimentado com uma tensão constante de 5 Volts e a tensão de saída apresentou valores variando de $511,41 \mathrm{mV}$ para uma altura 0 cm.c.a e $592,23 \mathrm{mV}$ para altura de 83 cm.c.a.

A análise da regressão linear dos pontos obtidos no ensaio é mostrada na Figura 7.

O cálculo do desvio padrão foi realizado somente entre as alturas de cada linha, e posteriormente foi calculado um desvio destes dados onde o valor obtido foi de 0,539 . Com este valor e pela análise do gráfico pode-se observar que a diferença entre as alturas real e calculada permanecem em uma mesma faixa.

Com base na regressão obtida, observa-se que o sensor responde linearmente ao conteúdo de água na coluna, pois apresenta um coeficiente de representatividade de 99,93\%. E comparando os resultados obtidos através da Equação 01, fornecida pelo fabricante e usando as propriedades da água a temperatura de operação, percebe-se que é necessária a calibração do equipamento. Desta forma, o sensor se apresenta adequado para utilização na bancada de controle de processos.

\section{Determinação da curva de calibração do sensor de vazão}

Na calibração do sensor de fluxo, foram obtidos os dados da Tabela 2, onde se pode observar os valores obtidos com doze pontos experimentais com pressão constante de 83 cm.c.a. A variação de vazão foi feita mudando o ângulo de abertura da válvula elétrica. Para cada linha da tabela a vazão foi mantida constante por quatro minutos e durante esse período se coletou dados a cada segundo durante 240 segundos, ou seja quatro minutos. Desses quatros minutos pegou-se os dados dos dois minutos centrais e fez-se a sua média para obter os dados que são apresentados na Tabela 2.

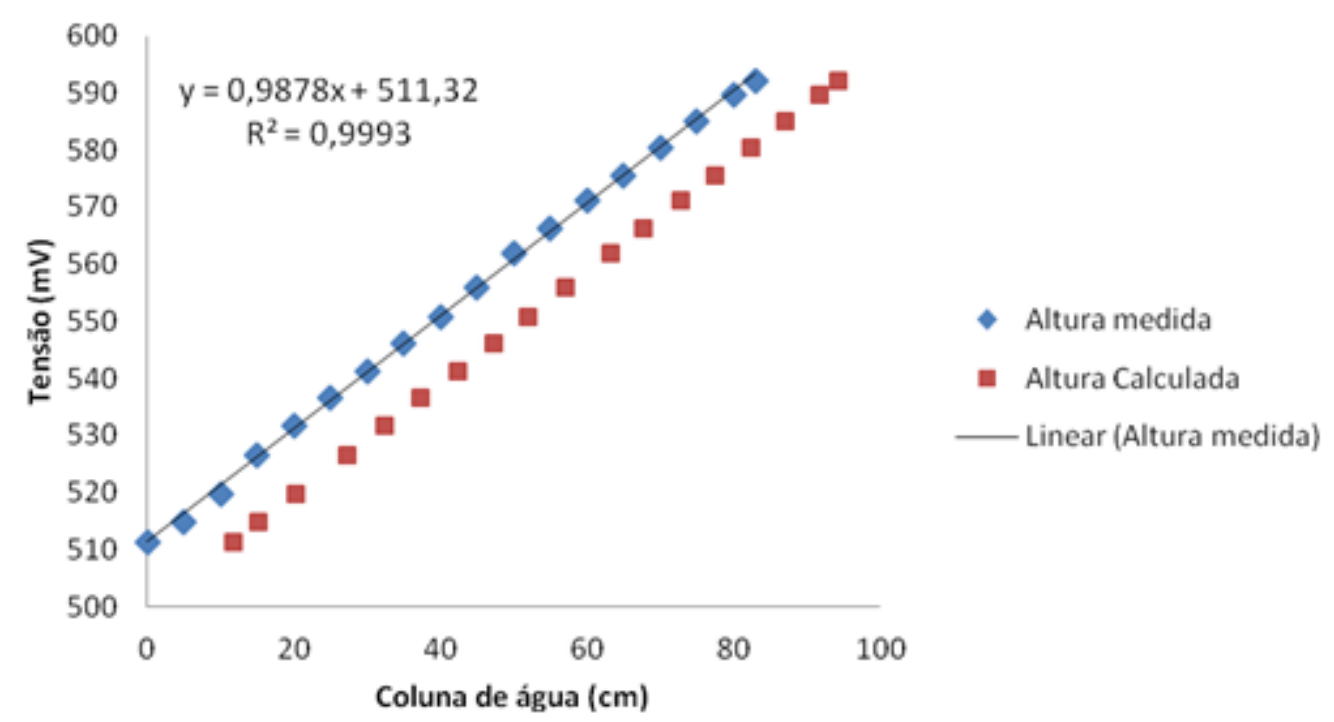

Figura 7. Curva de calibração do sensor de pressão, tensão de saída do sensor (mV) em função da coluna de líquido (coluna de água $(\mathrm{cm})$ ).

ENGEVISTA, V. 18, n. 2, p. 280-293, Julho 2016. 
O sensor de vazão foi alimentado com uma tensão constante de 5 Volts e a frequência dos pulsos apresentou valores variando de $2,15 \mathrm{~Hz}$ para uma vazão 0,281 L.min ${ }^{-1}$ e $53 \mathrm{~Hz}$ para vazão de 7,264 L. $\mathrm{min}^{-1}$.

A análise da regressão linear dos pontos obtidos no ensaio é mostrada na Figura 8.

Com base na regressão linear dos dados, observa-se que o sensor responde linearmente a vazão, pois apresenta um coeficiente de representatividade de 99,9\%. E comparando os resultados obtidos com a Equação 03, fornecida pelo fabricante e usando as propriedades da água a temperatura de operação, percebe-se que é necessária a calibração do equipamento. Desta forma, o sensor se apresenta adequado para utilização na bancada de controle de processos com reprodutibilidade.

Tabela 2. Dados obtidos através dos sensor Contech modelo YF-S201 e Equação 03, para o controlador de fluxo instalado no módulo experimental de controle de nível.

\begin{tabular}{cccc}
\hline $\begin{array}{c}\text { Frequência YF-S201 } \\
(\mathbf{H z})\end{array}$ & $\begin{array}{c}\text { Vazão Medida } \\
(\mathbf{L . m i n}-\mathbf{1})\end{array}$ & $\begin{array}{c}\text { Vazão calculada * } \\
\left(\text { L.min }^{-1}\right)\end{array}$ & $\begin{array}{c}\text { Desvio Padrão } \\
\text { entre as Vazões }\end{array}$ \\
\hline 0,00 & 0 & 0 & 0 \\
2,15 & 0,281 & 0,287 & 0,003897 \\
13,00 & 1,596 & 1,733 & 0,097093 \\
20,40 & 2,595 & 2,72 & 0,088345 \\
26,69 & 3,405 & 3,559 & 0,108966 \\
32,69 & 4,254 & 4,359 & 0,074255 \\
40,25 & 5,305 & 5,367 & 0,043324 \\
44,18 & 5,860 & 5,891 & 0,021851 \\
47,46 & 6,326 & 6,328 & 0,001265 \\
48,91 & 6,532 & 6,521 & 0,007819 \\
50,68 & 6,799 & 6,758 & 0,029337 \\
51,55 & 6,921 & 6,873 & 0,033779 \\
53,00 & 7,264 & 7,067 & 0,139611 \\
\hline
\end{tabular}

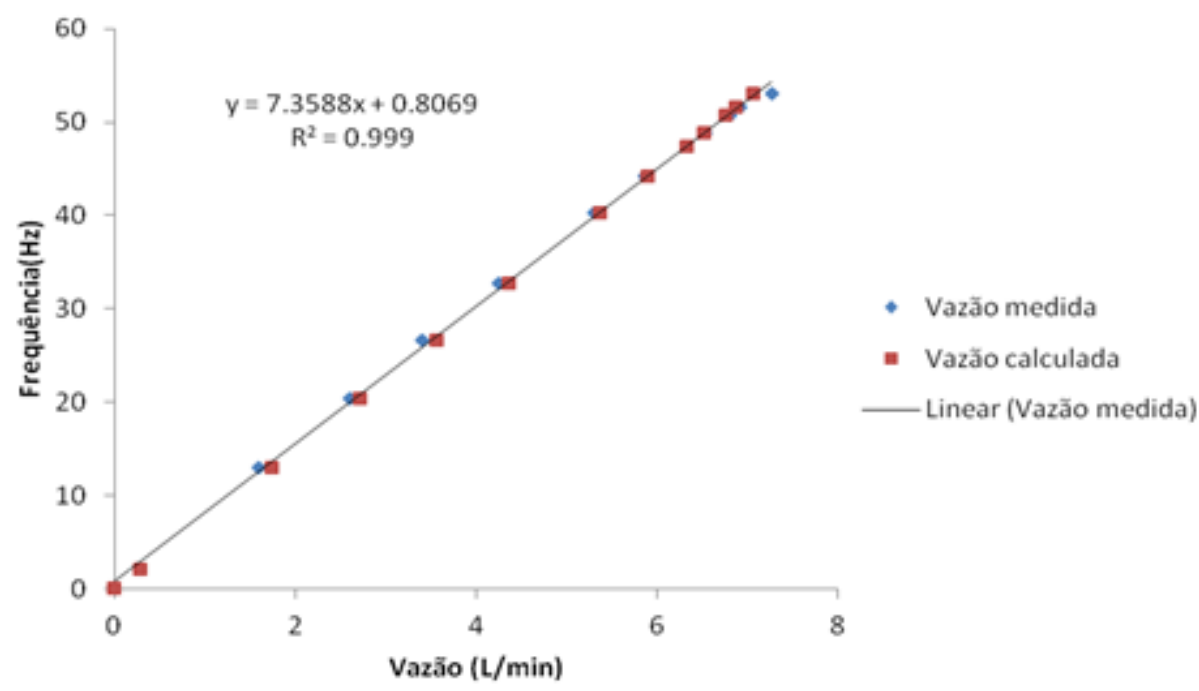

Figura 8. Curva de calibração do sensor de fluxo, frequência $(\mathrm{Hz})$ em função da vazão $\left(\mathrm{L} \cdot \mathrm{min}^{-1}\right)$. 


\section{Dependência da vazão proporcionada pela válvula esfera.}

Analisou-se a vazão de saída da válvula esfera em relação a sua abertura, pois não se espera um comportamento linear para a mesma, para isso manteve-se a pressão da coluna de água constante, e variou-se a abertura da válvula de 20 graus que foi a abertura que o sensor de fluxo modelo YF-S201 começou a responder até 90 graus que é a sua abertura máxima. A Tabela 3, apresenta os dados experimentais obtidos.

A Figura 9, apresenta a curva obtida para a abertura da válvula esfera em relação à vazão, pode-se observar que a pressão da coluna de água se torna constante, e portanto a vazão de operação do módulo de 7,3 L.min ${ }^{-1}$. Desta forma pode-se concluir que a vazão máxima de operação do módulo experimental projetado e instalado é de 7,3 L.min ${ }^{-1}$.

Tabela 3. Dados obtidos para a avaliação da válvula esfera utilizada no módulo experimental de controle de nível.

\begin{tabular}{cc}
\hline $\begin{array}{c}\text { Abertura } \\
\text { (Graus) }\end{array}$ & $\begin{array}{c}\text { Vazão } \\
\left(\text { L.min }^{-1}\right)\end{array}$ \\
\hline 20 & 0,281 \\
30 & 1,596 \\
35 & 2,595 \\
40 & 3,405 \\
45 & 4,254 \\
50 & 5,305 \\
55 & 5,860 \\
60 & 6,326 \\
65 & 6,532 \\
70 & 6,799 \\
75 & 6,921 \\
90 & 7,264 \\
\hline
\end{tabular}

Desta forma a vazão máxima de entrada no módulo que não pode exceder a 7,3 L.min ${ }^{-1}$, caso contrário o módulo não conseguirá dar vazão à alimentação, e consequentemente o módulo irá transbordar.

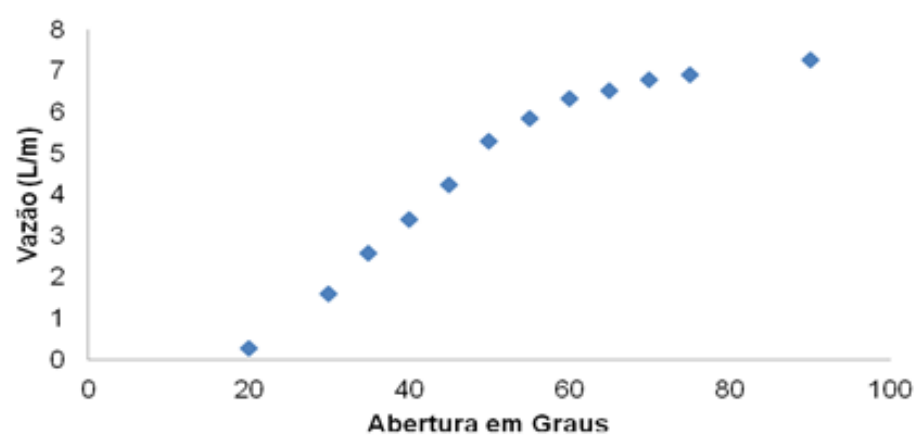

Figura 9. Curva obtida para a abertura da válvula esfera em graus, em função da vazão obtida em L. $\mathrm{min}^{-1}$ instalada no módulo experimental de controle de nível. 


\section{Funcionamento do módulo}

Para o módulo ser colocado em operação deverá se escolher um equipamento de controle que pode ser um Controlador Lógico Programável (CLP), placa Arduino ou outro controlador.

O funcionamento esperado para o módulo é que ao ser ligada a bomba, espera-se o regime ficar permanente e após isso é realizada uma perturbação na entrada do primeiro tanque, visualizase o comportamento da vazão e das colunas de líquido.

\section{Conclusões}

A execução do módulo de controle de nível proporcionou a experiência prática da engenharia. A calibração dos sensores de pressão e de fluxo, demonstrou que todos os sensores apresentam reprodutibilidade na faixa de operação a ser usada.

O módulo com os sensores calibrados está pronto para ser utilizado em experimentos de controle de processos, servindo para demonstrar o funcionamento de sensores analógicos e digitais, e atuadores na prática do ensino e da pesquisa.

\section{Lista de abreviaturas e símbolos}

$\begin{array}{cl}\text { bar } & \text { Unidade de pressão } \\ C & \text { Linguagem de programação } \\ C^{++} & \text {Linguagem de programação } \\ \text { CLP } & \text { Controlador Lógico Programável } \\ \text { cm.c.a } & \text { Centímetro de coluna d'água. } \\ g & \text { Aceleração da gravidade } \\ h & \text { Altura } \\ H z & \text { Hertz } \\ m m & \text { Milímetro } \\ m s & \text { Milissegundos } \\ m V & \text { Millivolts } \\ \text { PCV } & \text { Policloreto de polivinila } \\ \text { PID } & \text { Proporcional integral derivativo } \\ \text { PWM } & \text { Pulse Width Modulation } \\ \text { SAE 1020 } & \text { Aço de construção mecânica } \\ \text { VCC } & \text { Volts Corrente Contínua } \\ \rho & \text { Densidade }\end{array}$




\section{Referências}

ALBERNAZ, P. F.; ARAÚJO, V.; CARLOS, T. M. J.; MORAIS, J. S.; MORAIS, A. S. kit Didático para ensino da disciplina de controle digital de processos. X CEEL- Conferência de Estudos em Engenharia Elétrica. Universidade Federal de Uberlândia. Núcleo de Controle e Automação. Uberlândia, 2012.

BOJORGE, N. Unidade 06 - Sistemas de Medição de Nível, Departamento de Engenharia Química e de Petróleo- UFF, 2014.

MATHIAS, Artur Cardozo. Válvulas: Industriais, segurança, controle: tipos, seleção, dimensionamentos, Editora Artliber, São Paulo-SP, 2008.

NETO, J. G. da S.; NASCIMENTO, M.M. Instrumentação Virtual. Curso de especialização em instrumentação, automação, controle e otimização de processos contínuos. Universidade Federal da Bahia. Escola Politécnica. Bahia, 2007.

PINTO, F. da C. Sistemas de Automação e Controle. SENAI. Arcelor Brasil: CST. Espírito Santo, 2005.

RIBEIRO, M. A. Medição de Vazão, Fundamentos e Aplicação, $5^{a}$ edição, Salvador-BA, 2003

RIBEIRO, M.. A. Controle de processos teoria e aplicações. Tk treinamento e consultoria. $7^{\mathrm{a}}$ edição. Bahia, 2001.

SANTOS, A. Servomotores. Disponível em: <http://www.pictronics.com.br/downloads/apostilas/servomotores.pdf> acesso em 03 set 2014. Porto Alegre, 2007.

SCHMIDT, A. M. Controle de nível de liquido utilizando controlador lógico programável. 45f. Monografia (Graduação em engenharia de controle de automação). Universidade Federal de Ouro Preto - UFOP. Escola de Minas. Ouro Preto, 2008.

SEBORG, D. E.; EDGAR, T. F.; MELliCHAMP, D. A., Process Dynamics and Control, $2^{\circ}$ edição, 2003.

SEEED WIKI. G1/2 water flow sensor. Disponível em <http://www.seeedstudio.com/wiki/G1/2_Water_Flow_sensor> acesso em 05 set 2014. 2013.

SENAI. Instrumentação: Fundamentos de Controle de processo. Departamento Regional do Espírito Santo. CPM - Programa de Certificação do Pessoal de Manutenção. Espírito Santo, 1999a.

SENAI. Instrumentação básica II: Vazão, Temperatura e Analítica. Departamento Regional do Espírito Santo. CPM - Programa de Certificação do Pessoal de Manutenção. Espírito Santo, 1999b.

SMAR, Aspectos de Instrumentação \& Controle automático de processos, Centro de treinamento, <www.smar.com/brasil/PATS/downloads/Instrumentacao_\&_Controle.pdf> acesso em 15 de outubro 2014, Brasil, 2011. 